\title{
CONDITIONAL LEMPEL-ZIV ENCODERS
}

\author{
Marcelo S. Pinho and Weiler A. Finamore
}

\begin{abstract}
In this work, the concept of conditional LempelZiv encoder is presented. This concept is used to establish a variant of the Lempel-Ziv-Welch encoder $\left(L Z W^{-}\right)$. The practical performance of the new version is measured by using the Canterbury Corpus and it is compared to the performance of the $L Z W$. The new version has shown an improvement around $10 \%$ over the $L Z W$, when compressing the Canterbury Corpus.
\end{abstract}

Resumo - Este trabalho apresenta o conceito de codificador de Lempel-Ziv condicional. Este conceito é utilizado para estabelecer uma variante do codificador Lempel-Ziv-Welch ( $L Z W)$. O desempenho desta nova versão é avaliado, aplicando este compressor no Canterbury Corpus e comparado com o desempenho do $L Z H_{1}$. Esta nova versão apresenta um ganho de aproximadamente $10 \%$ sobre o $L Z I T$; quando aplicado no Canterbury Corpus.

Keywords: source coding, universal source coding, lossless data compression, string matching.

\section{INTRODUCTION}

In 1976 Lempel and Ziv proposed a complexity measure for finite sequences based on the string matching technique [1]. The results presented in that work showed that the string matching technique could be very useful to design universal source encoders. Using those concepts. Ziv and Lempel introduced two encoders. which are known as $L Z T T$ and $L Z 78[2,3]$. The practical importance of these encoders is due mainly to the relationship of their low complexity and good performance. Following those works, many variants were proposed, some of them widely used in practice [4].

Let $\mathcal{A}$ be a set of cardinality $c$ and let $\mathcal{A}^{\infty}$ denote the set of all one-sided infinite sequences. Let $p$ be a probability measure in $\mathcal{A}^{\infty}$. An information source is defined as a pair ( $\left.\mathcal{A} . p\right)$, where $\mathcal{A}$ is called the source alphabet. The semi-infinite sequence of random variables output by the source, drawn according to $p$, is denoted by $X_{1}^{\infty}=X_{1} \cdot X_{2} \ldots$

Let $\mathcal{A}^{*}$ be the set of all finite sequences of symbol drawn from $\mathcal{A}$. A lossless encoder $C$ is a reversible transformation which maps any finite sequence $u \in \mathcal{A}^{*}$ (also called word) into a finite sequence of bits $C(u)$ (also known as codeword).

Let $X_{1}^{n}=X_{1} \ldots X_{n}$ be a finite random sequence obtained from $X_{1}^{x}$ and let $x_{1}^{n}$ denote a realization of $X_{1}^{n}$ (with the convention that for $n=0, x_{1}^{n}$ denotes the empty sequence). Let

This work was supported by CNPq - Brazil. This work was presented in part at the Simpósio Brasileiro de Telecomunicações. Gramado-RS, Brazil. 2000

Marcelo S. Pinho is with the Department of Electrical Engineering. UNESP, Guaratinguetá. SP. Brazil. Weiler A. Finamore is with the Center for Telecommunications Studies, PUC-Rio, RJ. Brazil. E-mails: mpinho@feg.unesp.br. weiler@cetuc.puc-rio.br $|u|$ denote the length of the sequence $u$, that is, the number of symbols in $u$. Let $\mathcal{A}^{n}$ be the set of all length $n$ sequences.

Source coding theory aims at minimizing $E\left[\mid C\left(X_{1}^{n}\right)\right]$, where $E$ denotes expectation. A fundamental result of the source coding theory establishes that for any lossless encoder $C$, it is true that $E\left[\left|C\left(X_{1}^{n}\right)\right|\right] \geq H\left(X_{1}^{n}\right) \geq n H(\mathcal{A}, p)$, where $H\left(X_{1}^{n}\right)$, the entropy of the random sequence $X_{1}^{n}$, is defined as

$$
H\left(X_{1}^{n}\right)=\sum_{x_{1}^{n} \in \mathcal{A}^{n}}-\operatorname{Pr}\left[X_{1}^{n}=x_{1}^{n}\right] \log _{2} \operatorname{Pr}\left[X_{1}^{n}=x_{1}^{n}\right]
$$

and $H(\mathcal{A}, p)$. the source entropy, is defined as

$$
H(\mathcal{A}, p)=\lim _{n \rightarrow \infty} \frac{H\left(\mathrm{Y}_{1}^{n}\right)}{n}
$$

Many lossless encoders, Huffman Encoder and Arithmetic Encoder for instance, are such that $E\left[\left|C\left(X_{1}^{n}\right)\right|\right] \approx H\left(X_{1}^{n}\right)$ and the respective compression rate, $E\left[\frac{\left|C\left(X_{1}^{n}\right)\right|}{n}\right]$ (in bits per source symbol), converges to the source entropy, when $n$ goes to infinity [5]. These are encoders designed for sources with an specific probability measure $p$ which, whenever used to compress a source, with a different probability measure $q$, achieve perfromance that can be very poor. This mismatch poses strong limitations in practice. There is also the fact, that the source probability measure is just a model and it is unknown in many applications. Furthermore, there are many cases where an encoder is used to compress different kinds of data, drawn from different sources. A modem, for instance, uses the same encoder to compress images, text, programs, etc. The universal source coding theory is a branch of the source coding theory which addresses problems such as this. Its aim is to search for encoders which achieve good results for any source belonging to a given class of sources.

To find the best compression rate which can be attained by a lossless encoder, for a given class of sources, is a central question in universal source coding theory. An interesting result from this theory shows that it is possible to design lossless encoders that are optimal in the sense that their compression rates converge to the source entropy for many classes of sources. This result might lead us to the wrong assumption that there is no penalty in selecting a lossless universal encoder as opposed to using a lossless encoder whose design relies on the source probability measure. The problem with this assumption is that the optimality criterion which guarantees the convergence of the compression rate disregard the speed of convergence. The compression rate can converge to the source entropy, but if the convergency is too slow, the encoder will be useless, since in practice we deal with finite sequences. Therefore, a better criterion for an universal encoder is the asymptotic behavior of its compression rate with respect to a class of sources (instead of the limit of convergence $-H(\mathcal{A}, p))$. Using this criterion, it can be shown that 
there is no universal encoder which achieves the best performance $\left(\approx H\left(X_{1}^{n}\right)\right)$ for any source in a given class of sources.

In [3] it was shown that for any ergodic source $(\mathcal{A}, p)$, the $L Z 78$ compression rate, $\frac{\left|L Z 78\left(X_{1}^{n}\right)\right|}{n}$, converges to $H(\mathcal{A}, p)$. almost surely. In [6] it was shown that for any stationary source $(\mathcal{A}, p)$, the $L Z T 7$ compression rate, $\frac{\left|L Z T T\left(X_{1}^{n}\right)\right|}{n}$. converges to $H(\mathcal{A}, p)$, in probability. However, recently it was shown that the compression rates of these encoders do not converge as fast as it could, that is, there are universal encoders whose compression rates converge faster [7]. The disadvantage of these encoders, for which the compression rates converge faster, is their high computational complexity. Therefore, the $L Z 78$ and the $L Z 77$ are yet used in many applications.

The present work proposes a procedure to improve the performance of string match encoders. The rationale behind the proposition is briefly discussed next. Let $C$ be a sequential encoder via string matching (e.g. the $L Z 77$ or the $L Z 78)$. Given a realization $x_{1}^{n}, C$ parses the sequence in $m=m_{C}\left(x_{1}^{n_{2}}\right)$ phrases $s_{1}=x_{1}^{n_{2}}, s_{2}=x_{1+n_{2}}^{n_{3}}, \ldots, s_{m}=$ $x_{1+n_{m}}^{n_{1+m}}$, where each phrase $s_{i}=x_{i}^{j}, i<1+n_{i}$, that is, each phrase is a simple reproduction of a past string in the sequence. After that, the encoder $C$ encodes each phrase $s_{i}$, using a dictionary of strings, based on the past. In general, the well known string matching encoders, such as $L Z T i$ and $L Z 78$. do not use the knowledge of the phrase $s_{i-1}$ to encode the phrase $s_{i}$. In [8] it was developed an algorithm, based on the $L Z 77$ which uses the phrase $s_{i-1}$ to encode the string $s_{i}$. This encoder was called $L Z p$ and preliminary results have shown a good performance. In this work, it is shown a general description of the string matching encoders which use the knowledge of the phrase $s_{i-1}$ to encode the phrase $s_{i}$. which is called here Conditional Lempel-Ziv Encoders. This technique is used to improve the performance of the $L Z W$ encoder - the most used version of the $L Z 78$.

Section 2 presents the general model of a sequential string matching encoder and gives three examples, $L Z 77, L Z 78$. $L Z M$. The general description of the Conditional LempelZiv is given in Section 3. Section 4 is devoted to practical implementations and their results. Section 5 presents the conclusion.

\section{SEQUENTIAL STRING MATCHING ENCODER}

A sequential string matching encoder uses a dictionary of strings, derived from the part of sequence which was already encoded to encode the part of the sequence which remains to be coded. At the first step, the encoder establishes an initial dictionary which is used to compute the first phrase. The dictionary is then updated, at each step.

Figure 1 shows a generic step $i$ of a sequential string matching encoder. The sequence $x_{1}^{n_{i}}$ represents the part of the sequence $x_{1}^{n}$ which was encoded before step $i$. The part of sequence which remains to be coded is denoted by $x_{1+n_{i}}^{n}$. The box called "Dictionary" builds, using the sequence $x_{1}^{n_{i}}$, the updated dictionary $D_{i}$ which is used in step $i$. The box "Next Phrase" establishes the phrase $s_{i}=x_{1+n_{i}}^{n_{1+i}}$ which is

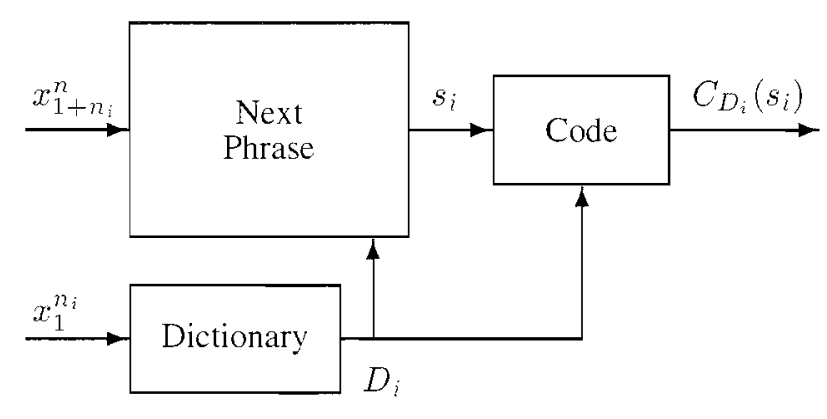

Figure 1. Sequential string matching encoders.

parsed off the sequence $x_{1+n_{i}}^{n}$ in step $i$. The box "Code" encode the phrase $s_{i}$ into its codeword $C_{D_{i}}\left(s_{i}\right)$, using a map which is dependent of the dictionary $D_{i}$. In general, the code $C_{D_{i}}$ is very simple and does not relies on any type of statistic measure. In fact. this is the simplicity that guarantees the low complexity of the encoder.

Following the general model, it is possible to define different sequential string matching encoders. An encoder is characterized when the four procedures below are specified.

1. Procedure to build the initial dictionary:

2. Procedure to build the dictionary $D_{i}$, which can either be an update procedure built upon $D_{i-1}$ and $x_{1}^{n_{1+i}}$, or a simple procedure, that uses just the sequence $x_{1}^{n_{1+i}}$.

3. Procedure to find the next phrase $s_{i}$, using the dictionary $D_{i}$;

4. Procedure $C_{D_{i}}$ to encode $s_{i}$.

The $L Z 77$ and the $L Z 78$ are examples of sequential string matching encoders. Therefore one can define these encoders just settling those four procedures. Several Lempel-Ziv variations are described next. In all cases the parameter $n_{1}$ (to be defined) is set equal to zero.

\section{$L Z 77$}

1. Initial Dictionary: $D_{1}=\mathcal{A}$.

2. Dictionary $D_{i}: D_{i}=\left\{u v: u=x_{j}^{k}, v \in \mathcal{A}, j \leq n_{i}, k \geq\right.$ $\left.n_{i}-1\right\}$.

3. Next Phrase: $s_{i}$ is the longest prefix of $x_{1+n_{i}}^{n}$ which belongs to $D_{i}$.

4. Code $C_{D_{i}}$ : Since $s_{i} \in D_{i}$, there is an integer $j \leq n_{i}$ such that $s_{i}=x_{j}^{k} v, v \in \mathcal{A}$. The phrase $s_{i}$ is encoded by an integer code of $k-j+1$, a simple code of $j$ plus a simple code (fixed length) of $v$.

It is important to point out that, since $u=\emptyset$ when $k$ assume the value $j-1$ the alphabet $\mathcal{A}$ is a subset of the dictionary $D_{i}=\left\{u v: u=x_{j}^{k}, v \in \mathcal{A}, j \leq n_{i}, k \geq n_{i}-1\right\}$. Since the range of $k-j$ is $\{-1,0,1, \ldots\}$, any non negative integer code can be used to encode the integer $k-j+1$ (e.g. the Elias Code [9]). The range of $j$ is $\{1, \ldots, i-1\}$. Therefore $j$ can be encoded using $\log _{2}(i-1)$ bits. The symbol $v \in \mathcal{A}$ needs $\log _{2} \alpha$ bits to be encoded. It is easy to see that in the first step, 
the integers $k-j+1$ and $j$ do not need to be encoded. In fact, it is easy to see that, since $D_{1}=\mathcal{A}, s_{1}=v$. Furthermore, if $k-j=-1$, then the integer $j$ does not need to be encoded, since $u=\emptyset$, the empty sequence, and therefore $s_{i}=v$.

$L Z 78$

1. Initial Dictionary: $D_{1}=\mathcal{A}$.

2. Dictionary $D_{i}: D_{i}=D_{i-1} \cup\left\{s_{i-1} v: v \in \mathcal{A}\right\}$.

3. Next Phrase: $s_{i}$ is the longest prefix of $x_{1+n_{i}}^{n}$ which belongs to $D_{i}$.

4. Code $C_{D_{i}}$ : Since $s_{i} \in D_{i}$, there is an older phrase $s_{j}, j<i$ such that $s_{i}=s_{j} v, v \in \mathcal{A}$. The phrase $s_{i}$ is encoded by a simple code of $j$ plus a simple code (fixed length) of $v$.

The range of $j$ is $\{0, \ldots, i-1\}$, therefore the integer $j$ can be encoded using $\log _{2} i$ bits. The symbol $v \in \mathcal{A}$ needs $\log _{2} a$ bits to be encoded. It is easy to see that in the first step. the integer $j$ does not need to be encoded. In fact, since $D_{1}=\mathcal{A}$. one has $s_{1}=v$.

There are many variants of $L Z 7 T$ and $L Z T 8$. One of the most famous is the variant of the $L Z T 8$ which was proposed in [10] and is called $L Z H$. The $L Z I I^{\circ}$ was designed to circumvent a weakness of the $L Z 78$. To understand this point more explicitly, consider the description of the $L Z T 8$. It is easy to see that, at step $i$, there are many phrases in the dictionary $D_{i}$ which never appeared in the past sequence $x_{1}^{n_{i}}$. Some of them, as a matter of fact. will rather likely, never appear again in the source sequence. In a text file, for example. when the letter $q$ appear, the $L Z 78$ includes a new phrases on $D_{i}$. Many of them, like $q u$ for instance, will obviously not be part of an ordinary text. The $L Z W^{\prime}$ get rid of this problem. avoiding the insertion of this type of phrases. Although this is problem that can be neglected in the asymptotic analysis, in practice it can not. In fact. simulation results using the $L Z I I$ have shown an improvement around $10 \%$ over the simulation results obtained with the $L Z 78$. The description of the $L Z I T$ is given bellow.

\section{$L Z H^{\circ}$}

1. Initial Dictionary: $D_{1}=A$

2. Dictionary $D_{i}$ : $D_{i}=D_{i-1} \cup\left\{s_{i-1} v^{\prime}: v\right.$ is the first symbol of $\left.s_{i}\right\}$.

3. Next Phrase: $s_{i}$ is the longest prefix of $x_{1+n_{i}}^{n}$ which belongs to $D_{i}$.

4. Code $C_{D_{i}}$ : Each element of $D_{i}$ has an index as follows. The elements of $\mathcal{A}$ is ordered from 0 to $\alpha-1$. The index of $s_{1} v$ is $\alpha$, the index of $s_{2} v$ is $c k+1$ and so on. The phrase $s_{i}$ is encoded by just encoding its index $i$ with a simple integer code.

The range of the index of $s_{i}$ in $D_{i}$ is $\{0, \ldots, \alpha+i-1\}$. Therefore the integer $j$ can be encoded using $\log _{2}(\alpha+i)$ bits.
The following examples illustrates the work of the encoders. The source sequence to be compressed is considered to be $x_{1}^{n}=010001101100$.

\section{Example $1 L Z 77$}

$$
\begin{aligned}
& \text { l. } D_{1}=\{0,1\} \text {, } \\
& s_{1}=0, k-j=-1, j=0, v=0 \\
& C_{D_{1}}\left(s_{1}\right)=0 \text {. } \\
& \text { 2. } D_{2}=D_{1} \cup\{0 v, 01 v, \ldots, 010001101100 v\} \\
& s_{2}=1 k-j=-1, j=0, v=1 \\
& C_{D_{2}}\left(s_{2}\right)=110,1 \text {. } \\
& \text { 3. } D_{3}=D_{2} \cup\{1 v, 10 v, \ldots, 10001101100 v\} \\
& s_{3}=00, k-j=0, j=1, v=0 \\
& C_{D_{3}}\left(s_{3}\right)=111,0,0 \text {. } \\
& \text { 4. } D_{4}=D_{3} \cup\{00 v, 000 v, \ldots, 0001101100 v\} \cup \\
& \cup\{00 v, 001 v, \ldots, 001101100 v\} \\
& s_{4}=011, k-j=1, j=1, v=1 \\
& C_{D_{4}}\left(s_{4}\right)=100110,11,1 . \\
& \cup\{11 v, \ldots, 1101100 v\} \cup \\
& \cup\{1 v, \ldots, 101100 v\} \\
& s_{5}=01100, k-j=3, j=5, v=0 \\
& C_{D_{5}}\left(s_{5}\right)=1011100,100,0 \text {. }
\end{aligned}
$$

\section{Example $2 \quad L Z 78$}

1. $D_{1}=\{0,1\}$,

$s_{1}=0$.

$C_{D_{1}}\left(s_{1}\right)=0$.

2. $D_{2}=D_{1} \cup\{00,01\}$

$s_{2}=1$

$C_{D_{2}}\left(s_{2}\right)=0.1$.

3. $D_{3}=D_{2} \cup\{10,11\}$

$s_{3}=00$,

$C_{D_{3}}\left(s_{3}\right)=01,0$.

4. $D_{4}=D_{3} \cup\{000,001\}$

$s_{4}=01$

$C_{D_{4}}\left(s_{4}\right)=01,1$.

5. $D_{5}=D_{4} \cup\{010.011\}$

$s_{5}=10$,

$C_{D_{5}}\left(s_{5}\right)=010.0$.

6. $D_{6}=D_{5} \cup\{100,101\}$

$s_{6}=11$.

$C_{D_{6}}\left(s_{6}\right)=010.1$.

7. $D_{i}=D_{6} \cup\{110,111\}$

$s_{i}=00$.

$C_{D_{i}}\left(s_{7}\right)=001.0$.

Example $3 \mathrm{LZH}^{-}$

I. $D_{1}=\{0,1\}$,

$s_{1}=0$

$C_{D_{1}}\left(s_{1}\right)=0$.

2. $D_{2}=D_{1} \cup\{01\}$

$s_{2}=1$,

$C_{D_{2}}\left(s_{2}\right)=01$.

3. $D_{3}=D_{2} \cup\{10\}$

$s_{3}=0$

$C_{D_{3}}\left(s_{3}\right)=00$. 


$$
\begin{aligned}
& \text { 4. } D_{4}=D_{3} \cup\{00\} \\
& s_{1}=00 \text {, } \\
& C_{D_{4}}\left(s_{4}\right)=100 . \\
& \text { 5. } D_{5}=D_{4} \cup\{001\} \\
& s_{5}=1 \text {, } \\
& C_{D_{5}}\left(s_{5}\right)=001 . \\
& \text { 6. } D_{6}=D_{5} \cup\{11\} \\
& s_{6}=10 \text {, } \\
& C_{D_{6}}\left(s_{6}\right)=011 . \\
& \text { 7. } D_{7}=D_{6} \cup\{101\} \\
& s_{7}=11 \text {. } \\
& C_{D_{\overline{7}}}\left(s_{7}\right)=110 \text {. } \\
& \text { 8. } D_{8}=D_{\bar{\tau}} \cup\{110\} \\
& s_{8}=00 \text {, } \\
& C_{D_{8}}\left(s_{8}\right)=0100 .
\end{aligned}
$$

\section{CONDITIONAL LEMPEL-ZIV}

It becomes evident from the encoders description presented that the code $C_{D_{i}}$ disregard the correlation tha may exist between the phrase $s_{i-1}$ immediately preceding $s_{i}$ and the phrase $s_{i}$. The phrase $s_{i}$ is encoded as if it were independent of $s_{i-1}$, such as in a memoryless model. It is true that the dictionary structure somehow build a context model for each symbol of $s_{i}$, as pointed out by Langdon [11]. A close examination of this structure reveals however that the initial portion of $s_{i}$ is modelled with a short context, which can be poor for the source model. An example of this poor choice is the context of the first symbol of $s_{i}, x_{1+n_{i}}$ the context is the empty sequence. Since the length of $s_{i}$ grows indetinitely, the influence of this bad effect vanishes in the limit when $n$ goes to infinity. When dealing with finite sequences this problem can be relevant yielding to significant performance degradation.

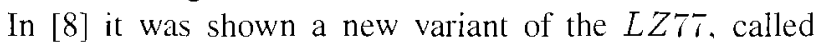
$L Z p$, which uses the phrase $s_{i-1}$ to build the code $C_{D_{i}}$. The $L Z p$ fix an order $k$ (related to the context length) and the phrase $s_{i}$ is such that $\mathcal{L}_{k}\left(s_{i-1}\right) s_{i}$ is the longest prefix of $\mathcal{L}_{k}\left(s_{i-1}\right) x_{1+n_{i}}^{n}$ which belongs to $D_{i}$. where $\mathcal{L}_{k}\left(s_{i-1}\right)$ are the $k$ last symbols of $s_{i-1}$. The code of $s_{i}$ is based on a subset $D_{i}\left(\mathcal{L}_{k}\left(s_{i-1}\right)\right)$ of $D_{i}$, which is the set of all sequences in $D_{i}$ which starts with $\mathcal{L}_{k}\left(s_{i-1}\right)$. Practical results have shown a good improvement. In fact. the result of the $L Z p$ for the Canterbury Corpus is one of the best in the literature. as reported in [8].

Although the idea of conditional Lempel-Ziv has been introduced in [8] as a variant of the $L Z \tau \pi$, the straightforward application of this idea to the $L Z 78$ will not be efficient [12]. The problem resides with the dictionary structure. Notice that the phrase $s_{i}$ produced by $L Z p$ tends to be shorter than the equivalent phrase produced by the original $L Z T \zeta$. The phrase $s_{i}$ is encoded using just a subset of $D_{i}$, and for this reason the corresponding codeword will also be smaller. Furthermore, the dictionary update procedure will not be affected by this modification.

The analysis of a straightforward conditional $-L Z \overline{7} 8$ leads to a different picture. As with the $L Z p$, a straightforward conditional $-L Z 78$ will tend to reduce the phrase $s_{i}$ and its corresponding codeword. However, in this case, the update of $D_{i}$ will be affected. This statement can be understood by noticing the fact, that the $L Z 78$ dictionary is updated by appending the phrases $s_{i} v, v \in \mathcal{A}$ and that if $s_{i}$ is reduced, the dictionary will be updated with reduced phrases. Even though the conditional technique yield an improvement in the performance at step $i$. the modification in the update procedure will tend to reduce the performance of the next steps. Therefore. a straightforward conditional- $L Z 78$ will not be effective.

To avoid this difficulty, by setting the value of the order $k$ to a value that maximizes the length of $s_{i}$ which turns out to be the same procedure used in a version of $L Z 78$, [13] called multi-match Lempel-Ziv, dubbed $m m L Z$.

The version $m m L Z$ was proposed to improve the parsing procedure of $L Z \mathrm{IT}$. In fact, from the description of the $L Z 78$ (and also of the $L Z M$ ) it is easy to see that the codeword length $C\left(x_{1}^{n}\right)$ increases with the number $m$ of parsed phrases. Therefore, if exist a procedure that can reduces $m$, then the performance of the encoder will be improved. In [14] it was shown that the procedure which finds the optimal parsing (with lowest number of phrases) for the $L Z 78$ is NPcomplete, a result which also holds for the $L Z W$. In [15] it is presented a procedure to compute the optimal parsing, when the dictionary of phrases is fixed - which is not the case of the $L Z 78$ and $L Z W$ encoders. The $m m L Z$ is briefly described next.

At each step $i$, the $m m L Z$ searches the pair $\left(s_{i}, s_{i+1}\right)$ in $D_{i} \times D_{1+i}$ which is the longest prefix of $x_{1+n_{i}}^{n}$. Notice that $s_{i}$ is not anymore. necessarily, the longest prefix of $x_{1+n_{i}}^{n}$ which belongs to $D_{i}$. In fact, sometimes it is interesting to reduce the phrase $s_{i}$ to find a longer $s_{1+i}$ such that the pair $s_{i} s_{1+i}$ is better than the pair established by the original encoder. However. it is obvious that the gain of $m m L Z$ is not obtained by reducing $s_{i}$, but by enlarging $s_{1+i}$. This enlargement is achieved by starting the phrase $s_{1+i}$ in a symbol $x_{j}$ different from $x_{1+n_{i+1}}, j \leq 1+n_{i+1}$. The idea of conditional Lempel-Ziv can therefore. be used to improve the $m m L Z$. Let $s_{i}$ be kept as in the original encoder and the let $s_{1+i}$ be such that $s_{i} s_{1+i}$ can be written as a concatenation of a pair $\left(\mathcal{F}_{j}\left(s_{i}\right), \mathcal{L}_{k}\left(s_{i}\right) s_{1+i}\right) \in D_{i} \times D_{i}$, where $\mathcal{F}_{j}\left(s_{i}\right)$ is the first synibols of $s_{i}$ such that $\mathcal{F}_{j}\left(s_{i}\right) \mathcal{L}_{k}\left(s_{i}\right)=s_{i}$. Thus the phrase $s_{1+i}$ can be encoded using a subset of $D_{1+i}$ which is composed by ail phrases which starts with $\mathcal{L}_{k}\left(s_{i}\right)$.

It is thus possible to establish new versions of the $L Z 78$ by using the concept of conditional Lempel-Ziv following the same ideas outlined in the above discussion. The version called $c L Z \mathrm{~W}^{\circ}$, built upon the $L Z \mathrm{WT}^{\mathrm{T}}$, with an illustrative example are presented next.

\section{cLZW}

1. Initial Dictionary $D_{1}=\mathcal{A}$.

2. Next Phrase: $s_{1}=x_{1}$. For $i>1, s_{i}$ is such that $s_{i-1} s_{i}$ is the longest prefix of $x_{1+n_{i-1}}^{n}$ which can be written as a concatenation of two phrases in $D_{i}$, that is, $s_{i-1} s_{i}=$ $s_{i-1}^{\prime} s_{i}^{\prime}$. where $\left(s_{i-1}^{\prime}, s_{i}^{\prime}\right) \in D_{i} \times D_{i}$.

3. Dictionary $D_{i}$ :

$D_{1+i}=D_{i} \cup\left\{s_{i}^{\prime} v: v\right.$ is the first symbol of $\left.s_{i+1}\right\}$. 
4. Code $C_{D_{i}}$ : From the NEXT Pharse procedure it can be seen that $s_{i}$ is a sufix of $s_{i}^{\prime}$. Let $u$ be the prefix of $s_{i}^{\prime}$ such that $s_{i}^{\prime}=u s_{i}$ ( $u$ can be the empty sequence). The phrase $s_{i}$ is encoded by the length of $u$, followed by the index of $s_{i}^{\prime}$ in a subset $D_{i}(u)$ of $D_{i}$ such that $D_{i}(u)=$ $\left\{y \in D_{i}: u\right.$ is a strict prefix of $\left.y\right\}$.

In the description of the $c L Z \mathrm{IT}^{\mathrm{T}}$, the Next Phrase procedure is placed before the Dictionary Update procedure to make the exposition easier. The range of the length $|u|$ of $u$ is less than $\left|s_{i-1}^{\prime}\right|$. Therefore it can be encoded using $\left\lceil\log _{2}\left|s_{i-1}^{\prime}\right|\right\rceil$ bits. Each subset $D_{i}(u)$ of $D_{i}$, for any $u \in D_{i}$ must be ordered and the index of $s_{i}^{\prime}$ can be encoded using $\left\lceil\log _{2}\left|D_{i}(u)\right|\right\rceil$ bits. To simplify the implementation of the encoder, the ordering of $D_{i}(u)$ can be established by using the ordering of $D_{i}$.

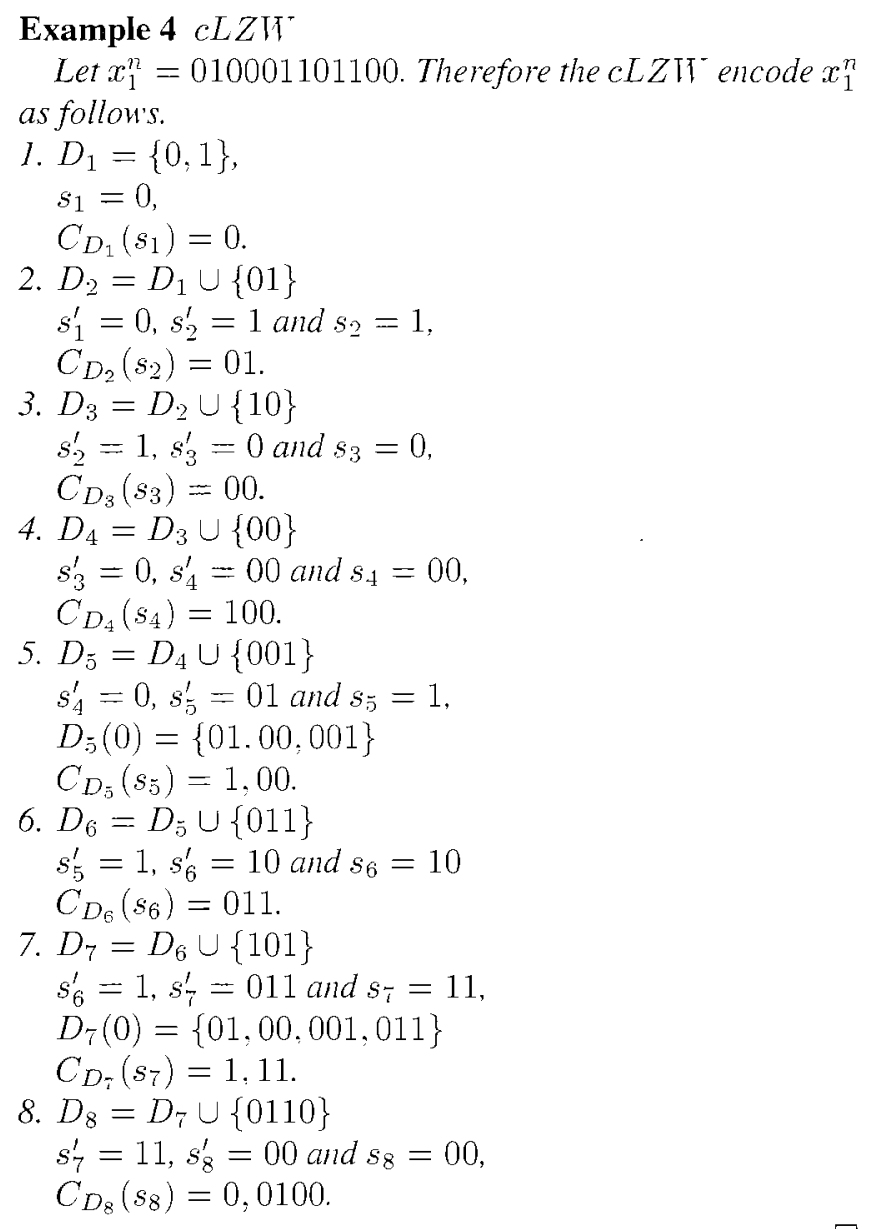

It can be seen from the description of the encoders that the length of the phrase $s_{i}$ tends to grow with $i$. Therefore, when the length $n$ of the input sequence $x_{1}^{n}$ is large, the length of the phrases $s_{i}$ can be large. The length of $s_{i}$ is an important parameter of the encoder because the $\mathrm{cLZ \textrm {NI } ^ { - }}$ computational complexity depends of it. In fact, the procedure to find the best pair $\left(s_{i-1}^{\prime}, s_{i}^{\prime}\right)$ is done by finding the longest prefix $s_{i-1}$ of $x_{1+n_{i-1}}^{n 2}$ followed by the next longest prefix $s_{i}^{\prime \prime}$. Once this task is completed, the phrase $s_{i-1}$ is reduced. by suppressing from it the last symbol. A search for a new longest prefix is then initiated. This procedure is repeated until the length of the phrase $s_{i-1}$ is equal to one. Then, the encoder compares the length of all pairs of phrases and chooses the longest pair.
Therefore, the complexity of the step $i$ of $c L Z W$ grows with the length of $s_{i-1}$. This complexity is not of much concern since the $L Z H$ complexity is very low, yet a small modification can be introduced in this encoder to bring the $c L Z W$ complexity even closer to the complexity of the $L Z W$.

As pointed out in the $c L Z W$ description, the $s_{i}^{\prime}$ can be written as $s_{i}^{l}=u s_{i}$. It can be see that the phrase $u$ is a sufix of $s_{i-1}$ and it is used as a context to encode the phrase $s_{i}$. As discussed before, the complexity of $c L Z W$ depends of the length of $s_{i-1}$, since the length of $u$ must be the best one among $\left\{0,1, \ldots,\left|s_{i-1}\right|-1\right\}$ and the search is exhaustively done. Therefore, if the length of $u$ is bounded, the complexity of the encoder will be reduced. This work denotes by $c L Z W / o$ the encoder that bounds the length of $u$ such that $|u| \leq o$. The description of this encoder is similar to the cLZTI and will be omitted.

\section{RESULTS}

In [16] the problem of establishing a good test set for a lossless encoder is discussed and a new test set is introduced. This new test set, called Canterbury Corpus, became popular and it has been used to measure the practical performance of many lossless encoders. The $c L Z W$ was implemented and its practical performance was measured, using the Canterbury Corpus. Table 1 presents the compression rate (in bits/symbol) obtained by the $L Z W$ and by the $c L Z I V$ when compressing the Canterbury Corpus.

\begin{tabular}{|c|c|c|c|}
\hline Files - $x_{1}^{n}$ & $\left|x_{1}^{n}\right|$ & $\frac{L Z W\left(x_{1}^{n}\right) \mid}{n}$ & $\frac{\left.c L Z W\left(x_{1}^{n}\right)\right]}{n}$ \\
\hline alice29.txt & 152089 & 3.26 & 2.91 \\
asyoulik.txt & 125179 & 3.51 & 3.20 \\
cp.html & 24603 & 3.68 & 3.24 \\
fields.c & 11150 & 3.56 & 2.99 \\
grammar.lsp & 3721 & 3.89 & 3.40 \\
kennedy.xls & 1029744 & 2.36 & 2.46 \\
lcet10.txt & 426754 & 3.02 & 2.64 \\
plrabn 12.txt & 481861 & 3.27 & 3.03 \\
ptt5 & 513216 & 0.97 & 0.97 \\
sum & 38240 & 4.20 & 3.72 \\
xargs.1 & 4227 & 4.42 & 4.02 \\
\hline \multicolumn{2}{|c|}{ Average } & 3.29 & 2.96 \\
\hline
\end{tabular}

Table 1. cLZW Results

As pointed out in Section 3, the complexity of the $c L Z W$ can be reduced, by bounding the length of the context. The encoder which bounds the context length is called $c L Z W / o$ where $o$ is the maximum length of the context. This encoder was implemented and was also used to compress the Canterbury Corpus. The results obtained are displayed in Table 2.

The results presented in Table 1 show that in the average, the $c L Z I T^{\circ}$ performance is $10 \%$ better than the $L Z W$ performance. Furthermore, from Table 2 it can be seen that the $c L Z \mathrm{IT}^{\circ} / O$ achieves a performance very close to the $c L Z W$ performance. Therefore, the penalty due to introduction of a bound for the context length can be neglected. This point is very interesting because it shows that the improvement of $10 \%$, obtained by the $c L Z W$, can be achieved using a version 


\begin{tabular}{|c|c|c|c|}
\hline Arquivos $-x_{1}^{n}$ & $\left|x_{1}^{n}\right|$ & $\frac{\left|c I Z \Pi / o\left(x_{1}^{n}\right)\right|}{n}$ \\
\cline { 3 - 4 } & & $O=2$ & $o=3$ \\
\hline alice29.txt & 152089 & 2.94 & 2.92 \\
asyoulik.txt & 125179 & 3.21 & 3.20 \\
cp.html & 24603 & 3.27 & 3.26 \\
fields.c & 11150 & 3.05 & 3.02 \\
grammar.lsp & 3721 & 3.47 & 3.43 \\
kennedy.xls & 1029744 & 2.44 & 2.46 \\
lcet10.txt & 426754 & 2.68 & 2.66 \\
plrabn I2.txt & 481861 & 3.05 & 3.03 \\
ptt5 & 513216 & 0.97 & 0.97 \\
sum & 38240 & 3.74 & 3.73 \\
xargs. 1 & 4227 & 4.05 & 4.04 \\
\hline \multicolumn{2}{|c|}{ Average } & 2.99 & 2.97 \\
\hline
\end{tabular}

Table 2. $c L Z W / o$ Results

whose complexity is very close to the $L Z W$.

\section{CONCLUSION}

This work presented the concept of conditional LempelZiv encoders. It showed how this technique can be used to improve the performance of the encoders based on string matching. A new version of the $L Z 78$, called $c L Z W$, was presented and its practical performance was measured by testing the encoder with Canterbury Corpus. Practical results have sfiown an improvement around $10 \%$ over the well known $L Z H$. Since the complexity of the $c L Z \mathrm{JW}^{-}$is greater than the $L Z W$ complexity, it was modified to keep the complexity near to the $L Z W$ complexity. The modified version, in short $c L Z W / o$, keeps a maximum context length of $o$. It was shown that the performance of the $c L Z \mathrm{H} \%$ approximates the performance of the $c L Z W$ for $o=2$. Therefore the improvement of $10 \%$ can be obtained by using a variant of the $L Z 78$ whose complexity is near to the $L Z I F$ complexity.

\section{REFERENCES}

[1] A. Lempel and J. Ziv, "On the complexity of finite sequences." IEEE Transactions on Information Theor?. vol. 22, pp. 75-81, 1976.

[2] J. Ziv and A. Lempel, "A universal algorithm for data compression," IEEE Transactions on Information Theorg, vol. 23. pp. 337-343, 1977.

[3] J. Ziv and A. Lempel, "Compression of individual sequences via variable-rate coding." IEEE Transactions on Information Theory, vol. 24, pp. 530-536, 1978.

[4] D. Hankerson, G. A. Harris. and P. D. J. Jr. Introduction to Information Theory and Data compression. Florida: CRC Press. 1998.

[5] T. M. Cover and J. A. Thomas, Elements of Information Theory. New York: Wiley, 1991.

[6] A. D. Wyner and J. Ziv, "The sliding-window lempel-ziv algorithm is asymptotically optimal," Proc. IEEE, vol. 82, pp. 872$877,1994$.

[7] F. M. J. Willems, Y. M. Shtarkov, and T. J. Tjalkens, "The context-tree weighting method: basic properties," IEEE Transactions on Information Theory, vol. 41, pp. 653-664, 1995.
[8] C. Bloom. "Lzp: a new data compression algorithm." Proc. of IEEE Data Compress Conference. 1996.

[9] P. Elias. "Universal codeword sets and representations of the integers." IEEE Transactions on Information Theon, vol. 21. pp. 194-203. 1975.

[10] T. A. Welch. "A technique for high-performance data compression." IEEE Computer. vol. 17, pp. 8-19. 1984.

[11] G. G. Langdon. "A note on the ziv-lempel model for compressing individual sequences." IEEE Transactions on Information Theory. vol. 29. pp. 284-287. 1983.

[12] M. S. Pinho. Codificadores Universais via Recorrência de Padróes para Fontes com Núnnero de Estados Finitos. PhD thesis, Pontifícia Universidade Católica do Rio de Janeiro, Departamento de Engenharia Elétrica. 1996.

[13] M. S. Pinho. W. A. Finamore. and W. A. Pearlman. "Fast multi-match lempel-ziv," Proc. IEEE Data Compress Conference. p. 545. 1999.

[14] S. D. Agostino and J. Storer. "On-line versus off-line computation in dynamic text compression." Infonnation Processing Letters, vol. 59. pp. 169-174. 1996.

[15] A. Hartman and M. Rodeh. "Optimal parsing of strings." Combinatorial Algorithms on Words, Springer-Verlag. A. Apostolico and Z. Galil. editors, pp. 155-167. 1985.

[16] R. Arnold and T. Bell, "A corpus for the evaluation of lossless compression algorithms." Proc. of IEEE Data Compress Conference, pp. 201-210. 1997.

Marcelo S. Pinho received the B.S.. M.S. and the D.S. degrees in Electrical Engineering from Pontifícia Universidade Católica do Rio de Janeiro, in 1994. 1996 and 2000. respectively. In 1998, he was a Visiting Scholar in the Image Processing Lab of the Rensselaer Polytechric Instiule. NY-USA. He is currenty a taculty member in the Department of Electrical Engineering of Universidade Estadual Paulista at Guaratinguetá. His research interests are on information theory, digital signal processing, and digital transmission.

Weiler A. Finamore was boin in Cisneiros. Minas Gerais. on October 16, 1944. He graduated in Electrical Engineering at Pontifícia Universidade Católica do Rio de Janeiro (PUC-Rio) in 1969. and got his MS and PhD degrees in Electrical Engineering from the University of Winsconsin. USA, in 1974 and 1978. respectively. He was a faculty member in the Department of Electrical Engineering of the Universidade Federal do Pará from 1970 to 1973 and during the 1978-79 academic year. Since 1979 he has been a faculty member in the Department of Electrical Engineering of PUC-Rio. working at its Center for Telecommunications Studies (CETUC). His research interests are on coding and information theory. digital image processing. and digital transmission. 